\title{
Carnets
}

Revue électronique d'études françaises de l'APEF

Deuxième série - 19 | 2020

Petite fabrique d'interprètes

\section{Duras Song}

\section{Maria do Rosário Girão Ribeiro dos Santos}

\section{(2) OpenEdition}

\section{Journals}

Édition électronique

URL : http://journals.openedition.org/carnets/11154

DOI : 10.4000/carnets. 11154

ISSN : 1646-7698

Éditeur

APEF

\section{Référence électronique}

Maria do Rosário Girão Ribeiro dos Santos, «Duras Song », Carnets [En ligne], Deuxième série - 19 | 2020, mis en ligne le 31 mai 2020, consulté le 23 décembre 2020. URL : http://

journals.openedition.org/carnets/11154; DOI : https://doi.org/10.4000/carnets.11154

Ce document a été généré automatiquement le 23 décembre 2020.

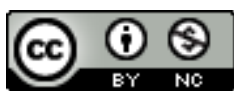

Carnets est mis à disposition selon les termes de la licence Creative Commons - Atribution - Pas d'utilisation commerciale 4.0 International. 


\title{
Duras Song
}

\author{
Maria do Rosário Girão Ribeiro dos Santos
}

1 Écrivaine, cinéaste et actrice, Marguerite Duras se positionne entre la continuité et la rupture, la modernité et la post-modernité, « au carrefour d'un certain néo-idéalisme, de l'existentialisme (...) de l'avant-garde» (Safranek, 1998: 59) et du «Nouveau Roman ». Sans prétendre à revisiter l'histoire de l' "École du regard ", tout en suivant un double critère chronologique et mécanique, il convient de souligner, toutefois, l'hétérogénéité visible (du point de vue littéraire) des nouveaux romanciers: si Nathalie Sarraute prône la recherche des états psychologiques, tapis dans le subconscient ${ }^{1}$, Alain Robbe-Grillet réfute cette vision spéléologique au profit de la description d'un univers qui, réduit à sa surface, semble obéir à des constructions photographiques $^{2}$ (ou photo-graphiques ?) et cinématographiques ${ }^{3}$. De même, tandis que l'univers de l'auteur de Les Gommes est celui des hommes parmi les objets, le domaine de Duras est celui des « hommes-objets » (Mauriac apud, 1993 : 131). Défenseur d'une acception non restrictive, mais plutôt large, du "Nouveau Roman", RobbeGrillet, à l'encontre de Jean Ricardou, s'empresse d'inclure Duras dans l'équipe des pionniers responsables de l'ouverture des voies nouvelles au roman nouveau, qui, selon lui, n'est pas une théorie, mais une recherche (Robbe-Grillet, 1963 : 114).

Car je persiste, moi, à inclure Duras dans le Nouveau Roman. Le critère de Ricardou selon quoi elle n'en fait pas partie parce qu'elle n'a pas voulu participer au colloque de Cerisy ne me semble pas pertinent. Marguerite Duras est quelqu'un d'extraordinairement personnel, obsédé par sa personnalité. Elle ne supporte pas d'être mélangée à quoi que ce soit. (...) Je suis contre toute idée restrictive de Nouveau Roman parce qu'une idée restrictive est contraire au projet même d'invention du roman. On ne peut pas dire : on va inventer le roman; et aussitôt voilà les règles de l'invention. Ce que j'ai réclamé pour le romancier, c'est justement la liberté. De sorte que considérer qu'untel correspond mal aux normes figées par Ricardou m'est antipathique. Bref, pour moi, Duras fait partie du Nouveau Roman. Ainsi que Beckett. (Micromégas, 1981 : 7).

2 La réplique de Ricardou à son confrère théoricien ne tarde pas à venir.

Une idée restrictive, celle qui limite un domaine rigoureux, ne saurait guère infliger, en soi, une quelconque entrave à l'invention. Il semble même avantageux de soutenir l'inverse : par la stricte définition qu'elle fournit d'un secteur précis, 
une idée restrictive peut permettre qu'enfin se réunisse, à partir de là, et de façon lucide, quelque peu autre chose. (...) une idée restrictive joue souvent le rôle, moins d'un frein que, diversement, d'un tremplin. (Ricardou, 1990 : 235-236)

$3 \mathrm{Au}$ sein et en marge de ces deux perspectives distinctes ${ }^{4}$ (la première élargie et la deuxième restreinte) le projet innovateur de Duras (qui a refusé toute étiquette), conciliant l'expérience vécue ou le référentiel / potentiel biographique et la réflexion sur le langage, débouche sur le questionnement de l'écriture, le besoin de la repenser et la renouveler. Ses définitions, par la négative, de l'acte d'écrire s'avèrent cruciales. De prime abord, écrire n'est pas, de son point de vue, le passage de l'être en puissance à l'être en acte ; ce n'est pas, non plus, le fait de raconter des histoires. Écrire est, plutôt, synonyme de déchiffrement de ce qui « est déjà là et qui déjà a été fait par vous dans le sommeil de votre vie » (Duras, 1987: 30)5. Cela étant, le fait d'écrire implique-t-il non seulement le dépeuplement de la vie ${ }^{6}$ (Duras, 2012 : 43), dont on est absent, mais aussi le "subissement» de l'inconnu que l'on porte en soi, susceptible d'atteindre à une certaine universalité. D'une part, on a le privilège de connaître l'état de la " dépersonne", de l' "im-personnalité " (Duras, 1999: 17), du renoncement à soi s'apparentant à une insuffisance de vie ${ }^{7}$; d'autre part, on est en mesure d'être envahi par le repeuplement, c'est-à-dire, l'invasion par des « êtres de papier » qui peuplent et enchantent l'existence moyennant l'expérience artistique. En outre, écrire ne réside pas dans le travail qui relève de l'effort (ces « affres de style » flaubertiens...), mais dans le non-travail, qui dissipe le labeur et cimente la genèse du livre à venir. Écrire consiste à raconter une histoire donnée et l'absence de cette même histoire, « une histoire qui passe par son absence » (Duras, 1987 : 31-32). D'où l'exigence de la solitude ${ }^{8}$ qui se hâte à réanimer l'écrit exsangue, à le vivifier et à se fondre et confondre avec lui. Dans ce contexte, écrire "c'est aussi ne pas parler. C'est se taire. C'est hurler sans bruit" (Duras, 2008: 28), c'est "à la fois se taire et parler» (Duras, $1995: 14$ ). Quant à la musique, elle est « le divin. (...) Il faut beaucoup chercher pour le trouver dans l'écrit, je l'ai trouvé (...) C'est la musique qui parle.» (Duras, 1987: 82). À son tour, le sujet qui écrit, à la recherche de la "bonne forme", devient le policier de soi-même: "On se flique en écrivant ", avoue Duras à Jean-Pierre Ceton. Cette image revient soit dans Écrire - «L'écrivain, alors il devient son propre flic » (Duras, $2008: 34$ ) -, soit dans Le ravissement de Lol V. Stein : «La police est en bas » (Duras, 1976 : 187). D'ailleurs, ce qu'on écrit « c'est une sorte de durée, de durée constante, toujours égale, qui est celle de la mer, celle du temps qui passe, de l'été, de cette espèce de temps (...) tragique, de l'été » (Duras, $2012:$ 59).

\section{Le bal estival}

4 Cette durée estivale est rythmée par le bal, espace par excellence de la rencontre, tendant vers l'épanouissement et/ou le refoulement du désir, assouvi ou inassouvi, puisque l'amour, inavouable, s'avère, dans la plupart des cas, impossible. Dans Le ravissement de Lol V. Stein, c'est le bal, au Casino Municipal de T. Beach, qui déclenche la folie de la protagoniste homonyme, née à $\mathrm{S}$. Tahla, délaissée par son fiancé, Michael Richardson, pour une femme mariée, Anne-Marie Stretter. Différemment de la psychologie ordinaire sous-jacente à la «triangulation» conventionnelle - «(..) triangulation dont l'aurore et eux deux sont les termes éternels» (Duras, 1976:47) -, cette dépossession provoque moins de la peine que de la fascination : «Je n'ai plus aimé mon fiancé dès que la femme est entrée. » (ibid. : 137). Tremblant et frémissant au loin, 
dans les recoins de la mémoire d'une femme abandonnée, cet épisode sera revisité, dix ans plus tard, par Lol qui, tellement emportée, une décennie auparavant, dans le spectacle de son bien-aimé et d'une femme habillée en noir, ne souffre pas d'être trahie : «- Quand je dis que je ne l'aimais plus, je veux dire que vous n'imaginez pas jusqu'où on peut aller dans l'absence de l'amour.» (ibid.: 138). Dans ce pèlerinage rétrospectif à $\mathrm{S}$. Tahla", en particulier à la salle de bal « La Potinière », Lol « regarde par à-coups (...) peut (...) revoir bêtement ce qui ne peut pas se revoir. » (ibid. : 181).

Scène fondatrice, par sa prégnance symbolique, le bal est, également, évoqué dans India Song, à travers les voix ( 1 et 2 ) de deux jeunes femmes, atteintes de folie, après le décès de Anne-Marie Stretter, alias Anna Maria Guardi : «Michael Richardson était fiancé à une jeune fille de $S$. Thala. Lola Valérie Stein. Le mariage devait avoir lieu à l'automne. Puis il y a eu ce bal. Ce bal de S. Thala... » (Duras, 2006:15). La remémoration, qui va de pair avec une toponymie détentrice d'un sens musical (ibid.), est irriguée par l'air "India Song ", qui alterne soit avec les silences qui se font sentir, soit avec les danses qui se succèdent (blues, tangos, fox-trott), soit avec les discours interrompus des personnages. Il faut retenir, dans ce contexte, que le leitmotiv musical déclenche, pour Anne-Marie Stretter, un endolorissement inexprimable, étant donné que la musique «c'est difficile à exprimer » et qu'une « certaine douleur s'attache à la musique » (ibid. : 85).

6 Par l'intermédiaire d'une gradation croissante, on assiste, dans L'Amour, à la reprise obsédante du bal à $\mathrm{S}$. Thala, dont la musique parcourt un large spectre d'émotions inhérentes à la disparition d'un temps perdu à jamais. Trois personnages animent le récit : une femme, un homme et un troisième homme ${ }^{10}$, un marcheur, qui prononce une phrase que Duras considère « la plus belle phrase de sa vie » (Duras, $1987: 34$ ).

- Ici, c'est $S$. Thala jusqu'à la rivière. (...)

- Après la rivière c'est encore $S$. Thala. (Duras, 1994 : 20-21).

7 Ce nom de lieu primordial, presque mythique, est écrit trois fois consécutives, comme s'il s'agissait d'un refrain, par le voyageur, l'homme de l'hôtel, dans une lettre: «S. Thala S. Thala S. Thala. » (ibid. : 20-21). Quant à la femme, qui avoue être «la morte de S. Thala " (ibid. : 78), elle s'approprie, tendrement, le toponyme, avec lequel elle et l'homme fusionnent.

- S. Thala, mon S. Thala. (ibid. : 102).

- S. Thala, c'est mon nom. (ibid. : 62).

Bref, l'anagnorisis a lieu :

- Je suis venue vous voir pour ce voyage. (...)

- Je n'y suis jamais revenue depuis que j'étais jeune. (ibid. : 99).

Dix-sept ans écoulés, la reconnaissance s'étend à l'hôtel, dont l'entrée est vide, à la salle de bal, où le bal n'a plus lieu, à la piste de danse (dans laquelle on ne danse plus), au piano fermé (d'où les notes ne s'envolent pas) et aux tapis rangés au long des murs. La musique, qui chantait dans la mémoire, devient une "marche lente aux solennels accents » et une «danse lente, de bals morts, de fêtes sanglantes.» (ibid. : 37). En plus, elle fait pleurer et se confond avec «la musique des fêtes mortes de $\mathrm{S}$. Thala, les lourds accents de sa marche » (ibid. : 66). Comme dans une antichambre de la mort, les phrases suspendues, le discours apparemment décousu et l'emprunt des 'techniques' à la prose poétique façonnent la mélodie d'une géométrie narrative, ébauchant la figure du triangle inhérente à la "triangulation" et distillant à compte-gouttes, grâce à des 
intermittences musicales (euphoriques auparavant, dysphoriques maintenant), une trame diégétique savamment conçue.

\section{2. «Trianguler » en été}

10 Cette géométrie narrative-musicale, nourrie par la « mise en abîme ", par la structure répétitive que le triangle emblématise et par l'art du contrepoint, semble traverser tous les ouvrages durassiens. Dans L'Amour («structure première » que l'on retrouve dans les récits postérieurs, selon Ceton et Duras), le motif du triangle humain, à l'image du triangle musical, donne la sensation de ne pas se fermer (ouvrant son troisième côté au passant qui incarne le désir), sans, toutefois, se briser à jamais.

Le triangle se ferme avec la femme aux yeux fermés. (...) Du fait de l'homme qui marche, constamment, avec une lenteur égale, le triangle se déforme, se reforme (...) Le glissement régulier du triangle sur lui-même prend fin : Il bouge. Il se met à marcher. (...) Le triangle se défait, se résorbe. Il vient de se défaire: en effet, l'homme passe, on le voit, on l'entend. (Duras, 1994 : 10-11)

11 Dans ce contexte, Lol Stein, après la mésaventure du bal, épouse le musicien Jean Bedford, qu'elle n'aime pas, et le trompe avec Jacques Hold, l'amant de sa meilleure amie Tatiana Karl, recréant une situation triangulaire où, loin d'être la victime, elle dicte désormais les règles du jeu. De même, le ravissement de son fiancé s'inscrit et est réécrit dans India Song, à travers le retour de Anne-Marie Stretter : d'une part, elle aime Michael Richardson et "n'est pas libre de cet amour »; d'autre part, le Vice-Consul de la France à Lahore, non nommé, "l'aime ainsi, dans l'amour de Michael Richardson » (Duras, 2006 : 96). Cette histoire d'amour, vécue dans les années 30 aux Indes, dans une ville surpeuplée aux bords du Gange, se croise, par l'évocation, avec la famine, la lèpre et les crématoires pendant la mousson de l'été et s'avère le contrepoint de la réception à l'Ambassade de France aux Indes, martelée par la $14^{e}$ Variation de Beethoven sur un thème de Diabelli et propice à la rumeur régnante. La musique, désignant trois variations de Beethoven sur un thème de Diabelli ou sur ce thème lui-même, traverse, également, Le Camion, film dont l'histoire est lue par Marguerite Duras et Gérard Depardieu : «Plan de l'écoute des deux, de la musique (thème de Diabelli). Ils attendent que cesse la musique... (...) Le thème de Diabelli arrive sur la musique, strident, et recouvre le déroulement du GÉNÉRIQUE. (Duras, 2014a : 24 et 70).

12 C'est, d'ailleurs, une sonatine de Diabelli qui, s'inscrivant dans l'incipit de Moderato Cantabile - mouvement au tempo modéré, entre allegro et andante -, structure deux axes sémantiques renvoyant à deux univers socialement distincts. Le premier, Moderato, dont l'acmé semble être la réception chez Anne Desbaresdes, dénote la norme, la routine et la modération : la leçon de piano, la mère aimante, l'enfant têtu, la maison bourgeoise et la promenade jusqu'au Boulevard de la Mer; le deuxième, Cantabile, dont le climax est la transgression 'plurielle' d'Anne dans le repas mondain, connote la déviance peu orthodoxe, la rupture et la passion virtuelle: l'espace du café que les ouvriers fréquentent, les rencontres avec Chauvin, les verres successifs de vin, la pulsion sexuelle et le désir (symbolisé par le magnolia) qui ne connaît pas la banqueroute. Ce deuxième récit tourne autour du récit-satellite (les micro-événements précédant les macro-événements), qui anticipe en révélant et révèle en condensant : un crime passionnel greffé sur l'indissociabilité d'Eros et de Thanatos, sur la conception d'une passion que la mort fascine et sur l'échec d'une expérience amoureuse 'réelle' et 
virtuelle. La «mise en abîme" est corroborée par les dernières tirades des protagonistes :

- Je voudrais que vous soyez morte, dit Chauvin.

- C'est fait, dit Anne Desbaresdes.

Anne Desbaresdes contourna sa chaise de telle façon qu'elle n'ait plus à faire le geste de s'y rasseoir. (...) Elle se retrouva face au couchant (...) (Duras, 1993: 123-124).

Une autre histoire de meurtre, encadrée dans une histoire ${ }^{11}$ de suicide, est bel et bien le thème de Dix Heures et demie du soir en été. Pendant les vacances, quatre touristes français arrivent à une petite ville espagnole, où un homme a tué sa femme et son amant. Ils seront contraints, à cause de l'orage, de passer la nuit dans un hôtel surpeuplé. À dix heures et demie du soir, Pierre, époux de Maria et père de Judith, devient l'amant de Claire, amie du couple, tandis que Maria essaye de sauver de la capture Rodrigo, le meurtrier: "Il s'agit d'un crime passionnel, d'un criminel d'occasion. » (Duras, 2015 : 68). Dans les méandres d'une double attente (la prison de l'assassin et le moment où Pierre et Claire s'aimeront), Maria ressent une fascination indéniable face à la passion (Seylas, 1963 : 23). En tombant amoureuse de Rodrigo, elle vit, par personne interposée et/ou par procuration, son propre drame, le suicide de son 'amant' correspondant à la fin de sa liaison conjugale : " - C'est la fin de notre histoire, dit Maria. Pierre, c'est la fin. La fin d'une histoire. (...) Je me tais. Mais, Pierre, c'est la fin. « (Duras, ibid. : 150).

14 Un couple se défait, un autre nait, comme c'est le cas dans L'après-midi de Monsieur Andesmas. Tout au long d'un lourd après-midi d'été, Monsieur Andesmas attend Michel Arc pour la construction d'une terrasse, dans une maison qu'il achète pour sa fille bienaimée Valérie, qui surplombera la vallée, le village et la mer. Cette attente, qui tourne au désespoir, n'est pas traduite par une durée extérieure, par le temps de l'horloge, par l'écoulement des heures et des minutes (comme dans Dix heures et demie du soir en été) ${ }^{12}$, mais plutôt par une durée intérieure, rendue évidente par la métaphore photographique (l'ombre et la lumière) et par la toile impressionniste (la « série » à la manière de Monet) que constitue l'ombre du hêtre perçue par le protagoniste.

Le soleil avait-il tourné ? (...) Un hêtre à quelques mètres de lui, le balaie de son ombre d'une noble grandeur, d'une imposante grandeur. (Duras, 2007 : 55).

L'ombre du hêtre est plus dense que celle du mur, elle est très vaste et il est en son milieu. (ibid. : 57).

Elle [la femme de Michel Arc] se trouvait hors de la portée de l'ombre du hêtre (...)

(ibid. : 62).

L'ombre du hêtre atteint maintenant le gouffre. Elle commence à s'y noyer. (ibid. :

79).

L'ombre avait maintenant gagné toute la plateforme. (ibid. : 86).

L'ombre commence à gagner les champs, elle s'approche du village. (ibid. : 92).

Deux espaces peuvent être signalés : en haut, dans la plateforme, l'attente de Monsieur Andesmas est à peine brisée par l'arrivée d'un chien roux, de la fille et de l'épouse de Michel Arc. En bas, le rectangle blanc de la place centrale (univers du désir de Valérie pour Michel Arc) constitue le scénario d'un bal, d'où s'envole une musique qui commence et recommence, disparait et réapparait - au rythme de laquelle Valérie et Michel Arc dansent -, contaminant le récit entier à travers la récurrence d'un refrain, repris intégralement ou partiellement, chanté par Valérie : «Quand le lilas fleurira mon amour / Quand le lilas fleurira pour toujours (...) Quand notre espoir sera là chaque jour / Quand notre espoir sera là pour toujours... »(ibid.: 16-22). L'explicit 
ouvert dit en ne disant pas, raconte sans raconter, défie le lecteur à appréhender l'accompagnement musical sous-jacent à la "triangulation » / dépossession : Monsieur Andesmas perd sa fille, qu'il aime passionnément, l'épouse de Michel Arc son mari, la fille son père. Et le récit termine avant que les personnages attendus n'apparaissent...

Cette disparition, synonyme d'absence de dénouement, traverse, aussi, Le Marin de Gibraltar. Un homme, le narrateur, qui veut changer de vie parce qu'il l'a gâchée, s'engage sur un yacht d'une Américaine (Anna), belle et riche, qui sillonne les mers, en quête, depuis trois ans, d'un légionnaire qui a déserté, recherché pour un assassinat, avec lequel elle a connu une période inoubliable de bonheur. Ce soi-disant récit de voyage, d'un continent à l'autre - de Sète à Tanger, de Tanger à Abidjan et d'Abidjan à Léopoldville -, pendant les vacances italiennes, au long duquel elle emmène sur son bateau un homme qui lui plait, s'achève, après maintes péripéties, sur le renoncement à un grand amour et sur la substitution progressive du narrateur par le marin... Dans la durée matérielle, qui mûrit le sentiment mutuel, la procrastination d'un aveu sentimental est comme que retardée par la sempiternelle absence du marin, et à la suite du voyage... à la recherche du marin ou d'un autre amour : « La mer fut très belle vers les Caraïbes. Mais je ne peux pas encore en parler. » (Duras, 1977 : 430).

En fait, et d'un ouvrage à l'autre, les motifs se répètent d'une façon obsédante: l'omniprésence de l'été et de la chaleur, l'oisiveté des longues journées et des vacances estivales (voir l'inscription de l'été dans les titres, comme c'est le cas de L'Été 80 et de Dix heures et demie du soir en été); l'écriture de la représentation, visuelle, et la représentation de l'image ${ }^{13}$; la liberté symbolisée par la mer, le port et la vedette ; le bal, scène matrice où se met en place la situation triangulaire ; la figure géométrique du quadrilatère, de l'espace rectangulaire, qui baigne, en général, dans la lumière et renvoie à la scénographie du désir ; la présence de l'enfant, tremplin pour la rencontre ou pour le dialogue cérémonieux et précieux : «L'enfant arriva tranquillement du fond du square et se planta devant la jeune fille. (...) Ce fut pour l'homme l'occasion d'engager la conversation. » (Duras, 1983: 9); le meurtre passionnel - récit-satellite dans Moderato Cantabile et Dix Heures et demie du soir en été - qui donne l'impression de s'ébaucher dans L'Homme assis dans le couloir : « Je vois que l'homme pleure couché sur la femme. Je ne vois rien d'elle que l'immobilité. Je l'ignore, je ne sais rien, je ne sais pas si elle dort.» (Duras, 2014c: 36); le cri, aux antipodes du silence, qui résonne dans Moderato Cantabile et dans L'Amour : « Le cri arrivait de là. " (Duras, 1994 : 28) ; le chien qui parcourt L'après-midi de Monsieur Andesmas - «C'était un chien roux, de petite taille. Il venait sans doute des agglomérations qui se trouvaient sur l'autre pente, (...)» (Duras, 2007: 9) et L'Amour: "Le voyageur (...) marche lentement, il va dans la direction qu'a prise le chien. » (Duras, $1994: 21)$; la toponymie, détentrice d'un sens musical (S. Thala), ainsi que l'onomastique significative (Valérie Stein et Valérie Andesmas); et le regard, mêlé à la passion, déclencheur d'un quelconque événement que l'on attend.

L'action se raréfie, se désintègre, au niveau sémantique et diégétique. Les silences prolifèrent, à l'instar des blancs et des points d'indétermination; les mots-trous envahissent le discours des personnages; les phrases incomplètes, qui ne sont pas jetées au hasard, fragmentent et brisent la linéarité textuelle, que le récit récupère ; quant au dialogue, il semble, apparemment, ne pas accomplir sa fonction de communication, mais plutôt son rôle d'agent de l'incommunicabilité ; la description se révèle créatrice et dynamique, contribuant à un « corps-écriture » énigmatique (Udris, 
1998 : 192-193). L'architecture devient mélodie : la structure répétitive, la présence de l'oralité, la "mise en abîme ", les refrains, l'art du contrepoint et le jeu d'échos et reprises lui confèrent une musicalité originale, renforcée par les références aux variations de Beethoven sur un thème de Diabelli, par les sonatines de Diabelli ou par les échos des airs chantonnés qui émanent du bal.

Il ne faut pas oublier, toutefois, la dimension politique et sociale de l'œuvre durassienne.

Je crois à l'utopie politique, c'est-à-dire je crois profondément au mouvement d'Allende (...) C'est l'utopie qui fait avancer les idées de gauche, même si elle échoue. 68 a échoué, ça a fait un pas en avant fantastique pour l'idée de gauche, ce qu'on a appelé longtemps l'exigence communiste, mais qui dans la conjoncture actuelle ne signifie plus rien du tout. (Duras, 2014b : 114).

Tout s'est tourné entre Trappes et Plaisir, c'est-à-dire, en somme, la capitale de l'émigration. Je ne sais pas combien ils sont, peut-être un million ou deux millions dans le coin (...) Je dois dire que les Portugais, dans les premiers temps, se sont sauvés des appartements qu'on leur assignait, pour aller retrouver leurs caravanes et leurs bidonvilles, parce que dans leurs bidonvilles ils étaient ensemble, le soir ils pouvaient manger ensemble, se réunir. Il y avait une véritable communauté dans les bidonvilles. (ibid. : 108).

En ce qui concerne les Portugais, il convient, aussi, de ne pas passer sous silence un petit extrait de Le Théâtre de l'amante anglaise :

Qu'elle [Marie-Thérèse Bousquet] avait pu partir avec un homme, un Portugais; les Portugais s'en fichent qu'elle soit sourde ou muette. Ils ne parlent pas le français. (Duras, 1991a : 22).

Cette note est, peut-être, la seule note dissonante de Duras Song ... qui passe des mots sur la page aux images sur l'écran.

\section{BIBLIOGRAPHIE}

BORGOMANo, Madeleine (1985). L'Écriture filmique de Marguerite Duras. Paris : Éditions Albatros, « Cinéma ».

DURAS, Marguerite (1976). Le Ravissement de Lol V. Stein. Paris : Gallimard, « Folio ».

DURAS, Marguerite (1977). Le Marin de Gibraltar. Paris : Gallimard, « Folio ».

DURAS, Marguerite (1983). Le Square. Roman. Paris : Gallimard.

DURAS, Marguerite (1987). La Vie matérielle. Paris : POL.

DURAS, Marguerite (1991a). Le Théâtre de l'amante anglaise. Paris : Gallimard, «L'Imaginaire ».

DURAS, Marguerite (1991b). L'Amant. Paris : Minuit.

DURAS, Marguerite (1993). Moderato Cantabile. Paris : Minuit.

DURAS, Marguerite (1994). L'Amour. Paris : Gallimard, « Folio ». 
DURAS, Marguerite (1995). C'est tout. Paris : P.O.L.

DURAS, Marguerite (1999). Dits à la télévision. Entretiens avec Pierre Dumayet. E.P.E.L., ag « Atelier ». DURAS, Marguerite (2006). India Song. Texte, théâtre, film. Paris : Gallimard, « L'Imaginaire ». DURAS, Marguerite (2007). L'Après-midi de Monsieur Andesmas. Paris : Gallimard, « L'Imaginaire ». DURAS, Marguerite (2008). Écrire. Paris : Gallimard, « Folio ». DURAS, Marguerite (2014a). Le Camion, suivi de Entretiens avec Michelle Porte. Paris : Minuit. DURAS, Marguerite (2014b). Entretiens avec Michelle Porte précédés de Le Camion. Paris : Minuit. DURAS, Marguerite (2014c). L'Homme assis dans le couloir. Paris : Minuit. CETON, Jean Pierre (2012). Entretiens avec Marguerite Duras. Paris : François Bourin Éditeur. LOIGNON, Sylvie (2001). Le Regard dans l'œuvre de Marguerite Duras. Circulez, y'a rien à voir. Paris : L'Harmattan.

MARGARIDO, Alfredo, Artur Portela FILHO (1962). o Novo Romance. Lisboa : Editorial Presença. MAURIAC, Claude (1958). « L'étouffant univers de Marguerite Duras », Le Figaro, 12-3-58, in « Moderato Cantabile et la presse française », apud Moderato Cantabile (1993). Paris : Minuit. RICARDOU, Jean (1967). Problèmes du nouveau roman. Paris : Seuil. « Tel Quel ». RICARDOU, Jean (1990). Le Nouveau Roman suivi de Les raisons de l'ensemble. Paris : Seuil. « Essais ». ROBBE-GRILLET, Alain (1963). Pour un nouveau roman. Paris : Minuit.

ROBBE-GRILLET, Alain (1981). « Survivre à sa mode », Micromégas. Rome : Éditions Bulzoni, n²0, apud RICARDOU, Jean (1990). Le Nouveau Roman suivi de Les raisons de l'ensemble. Paris : Seuil.

SAFRANEK, Ingrid (1998), « Texte des origines, origines du texte (de la recette de cuisine à l'art poétique ", in Catherine Rodgers et Raynalle Udris (org.). Marguerite Duras. Lectures plurielles. Amsterdam-Atlanta : GA, pp. 57-75.

SARRAUTE, Nathalie (1993). L'Ère du soupçon. Essais sur le roman. Paris : Gallimard.

SEYLAS, Jean-Luc (1963). Les Romans de Marguerite Duras. Essai sur une thématique de la durée. Paris : Archives des Lettres Modernes.

UDRIS, Raynalle (1998), « Réception et production du sens dans le texte durassien », in Catherine Rodgers et Raynalle Udris (org.). Marguerite Duras. Lectures plurielles. Amsterdam-Atlanta : GA, pp. 185-199.

\section{NOTES}

1. Voir la «Préface » de L'Ère du soupçon : «On commence maintenant à comprendre qu'il ne faut pas confondre sous la même étiquette la vieille analyse des sentiments, cette étape nécessaire, mais dépassée, avec la mise en mouvement de forces psychiques inconnues et toujours à découvrir dont aucun roman moderne ne peut se passer. » (Sarraute, 1993 : 10-11).

2. Par rapport à la photographie, Sylvie Loignon affirme qu'elle «n'est importante que dans la mesure où elle est absente et où elle pointe une absence. Elle navigue entre mémoire et oubli aux confins de la mort et de la folie. » (Loignon, 2001 : 96).

3. Après avoir écrit L'Amour, Marguerite Duras, qui conteste la spécificité des genres, renonce à la littérature pour se consacrer au cinéma. L'écriture de Duras, filmique, est visible dans le 
découpage des scènes, dans les gros plans, dans les prises de vue, dans la profondeur du champ et dans la lenteur du rythme.

4. Pour ce qui est des perspectives différentes des nouveaux romanciers, il faut souligner l'importance des réponses de Michel Butor, Claude Simon et Nathalie Sarraute à un questionnaire élaboré par Alfredo Margarido e Artur Portela Filho. Ces témoignages ont été recueillis dans l'ouvrage $O$ Novo Romance. Cela étant, et pour Michel Butor, le cinéma a rendu la mémoire plus visuelle; en ce qui concerne Claude Simon, le langage et l'écriture ne sont pas au service d'une quelconque signification, mais constituent la 'matière' première de l'œuvre; quant à Nathalie Sarraute, elle est d'avis qu'il faut considérer le roman comme étant un art, et non pas un mythe de distraction ou d'information. (Margarido, Filho, 1963 : 147-153).

5. Voir, aussi, la définition de l'écrit que donne Duras dans Écrire: «L'écrit ça arrive comme le vent, c'est nu, c'est de l'encre, c'est l'écrit, et ça passe comme rien d'autre ne passe dans la vie, rien de plus, sauf elle, la vie. » (2008:53).

6. «Il n'y a pas d'écriture qui vous laisse le temps de vivre ou bien il n'y a pas d'écriture du tout (...) Puisque ce que vous mettez dans le livre, ce que vous écrivez, ce qui sort de vous, ce qui en passe par vous plutôt, c'est ça en définitive le plus important de tout ce que vous êtes. » (Ceton, $2012: 44)$.

7. Voir, dans ce contexte, les réflexions de la narratrice de L'Amant : «Je crois que j'ai vaguement envie d'être seule, (...) Je vais écrire des livres. C'est ce que je vois au-delà de l'instant, dans le grand désert sous les traits duquel m'apparaît l'étendue de ma vie.» (1991b : 126).

8. Dans Écrire, Duras donne une définition magistrale de la solitude: "On ne trouve pas la solitude, on la fait. La solitude elle se fait seule. Je l'ai faite. » (2008:17).

9. Dans Le Ravissement de Lol V. Stein, le toponyme S. Thala est écrit d'une façon différente de celle que l'on trouve dans India Song et dans L'Amour [S. Tahla].

10. À ce propos, Duras avoue à Ceton : «Ce troisième homme, voyez-vous, qui marche le long de la plage, j'essaie toujours, toujours, de l'amener vers les autres. Je pense que c'est moi, l'homme qui marche, enfin c'est l'auteur. J'essaie toujours de l'amener dans la perte, dans la perte de la peur où sont les autres. » (Ceton, $2012: 28$ ).

11. Voir, à ce sujet, Ricardou, Jean, « L'histoire dans l'histoire » (1967: 171-189).

12. « Un peu plus de deux heures et demie (...) Il est dix heures (...) Midi moins vingt-cinq (...) Il est un peu plus de midi (...)» $(2015: 84,97,110$ et 118$)$.

13. L'image, pour Madeleine Borgomano, « saisit très souvent les personnages dans l'immobilité ; cette immobilité nous devient très sensible et nous pèse particulièrement au moment où elle est perçue par le témoin étranger » $(1985: 72)$.

\section{RÉSUMÉS}

Quoiqu'apparentée au "Nouveau Roman ", Marguerite Duras a toujours refusé toute étiquette. Franchissant les genres littéraires et glissant du récit poétique au texte filmique et théâtral, sa poétique originale est façonnée par la dialectique de l'expérience vécue et du langage contrôlée, le renouvellement de l'écriture questionnée, la raréfaction de l'action, la revendication d'une ouverture textuelle, la désintégration du sens, la métaphore photographique et l'omniprésence du regard. Quant à la musique, elle s'inscrit littéralement et métaphoriquement dans sa géométrie thématico-narrative : l'architecture musicale, la structure répétitive (renforcée par la 
mise en abyme), la scénographie du désir (la rencontre, le bal et la «triangulation »), l'art du contrepoint, la durée néfaste ou le tempo singulier et la présence obsédante de la mer et de l'été. Sous la canicule étouffante, pendant les vacances et au long des journées oisives, meublées par des silences, l'atmosphère devient irrespirable, sauf en ce qui concerne Duras Song

Although Marguerite Duras is often associated with the "Nouveau Roman ", she has always refused any label. Covering the literary genres and moving from poetic narrative to filmic and dramatic texts, her original poetics is shaped by the dialectics of live experience and controlled language, the renewal of questioned writing, the rarefaction of action, the claim of a textual opening, the disintegration of meaning, the photographic metaphor and the omnipresence of the gaze. As for music, it literally and metaphorically fits into its thematic-narrative geometry: the musical architecture, the repetitive structure (reinforced by the "mise en abyme»), the scenography of desire (the ball and the "triangulation»), the art of counterpoint, the duration or the singular tempo, and the presence of summer. Under the suffocating heat of long, idle days filled with silences, the atmosphere becomes unbreathable, except for Duras Song.

\section{INDEX}

Mots-clés : écrire, musique, bal, « triangulation », contrepoint

Keywords : write, music, ball, «triangulation », counterpoint

\section{AUTEUR}

\section{MARIA DO ROSÁRIO GIRÃO RIBEIRO DOS SANTOS}

Un. do Minho

rosario_santos[at]ilch.uminho.pt 